Bundesgesundheitsbl 2016 $59: 438-443$ DOI 10.1007/s00103-016-2314-y

Online publiziert: 15. März 2016

(c) The Author(s) 2016 . This article is available at SpringerLink with Open Access

CrossMark
Oliver Peters' · Isabella Heuser' - Lutz Frölich ${ }^{2}$ Eckart Rüther ${ }^{3} \cdot$ Otto Rienhoff ${ }^{3}$. Johannes Kornhuber ${ }^{4} \cdot$ Jens Wiltfang ${ }^{3} \cdot$ Wolfgang Maier $^{5}$

${ }^{1}$ Klinik für Psychiatrie und Psychotherapie, Charité Universitätsmedizin Berlin - CBF, Berlin, Deutschland

${ }^{2}$ Zentralinstitut für Seelische Gesundheit Mannheim, Mannheim, Deutschland

${ }^{3}$ Institut für Medizinische Informatik, Universitätsklinik Göttingen, Göttingen, Deutschland

${ }^{4}$ Psychiatrische und Psychotherapeutische Klinik, Universitätsklinikum Erlangen, Erlangen, Deutschland

${ }^{5}$ Klinik und Poliklinik für Psychiatrie und Psychotherapie, Universitätsklinikum Bonn, Bonn, Deutschland

\title{
Das Kompetenznetz Demenzen
}

\section{Erfolge und Ausblicke}

Das Kompetenznetz Demenzen (KND) wurde als eines der jüngsten Kompetenznetze der ersten Generation von 2002-2009 vom Bundesministerium für Bildung und Forschung (BMBF) über zwei Perioden zuzüglich Laufzeitverlängerung für die Durchführung und Beendigung der medikamentösen Therapiestudien gefördert (2002-2005; 2005-2008; Laufzeitverlängerung bis 2009). Die Struktur des geförderten Kompetenznetzes Demenzen untergliedert sich in die drei Bereiche Weiterentwicklung diagnostischer Instrumente einschließlich Neuropsychologie, Biomarker, Bildgebung und Genetik (E1), Therapiestudien bei kognitiven Störungen und Demenzen (E2) sowie Versorgungsforschung (E3). An der Arbeit des Kompetenznetzes Demenzen haben sich insgesamt 13 mehrheitlich psychiatrische Universitätskliniken mit ihren akademischen Gedächtnissprechstunden beteiligt. Das Kompetenznetz Demenzen existiert seit dem Ende der Förderung durch das BMBF als eingetragener Verein (KND e. V.) mit Sitz am Zentralinstitut für Seelische Gesundheit (ZI) in Mannheim und einer Geschäftsstelle an der Charité in Berlin. Sprecher des KND e.V. ist (seit 2006) Prof. Wolfgang Maier (Bonn) - vorher Prof. Fritz Henn (ZI), stellvertretender Sprecher ist Prof. Eckart Rüther (Feldafing). Weitere Vorstandsmitglieder sind: Prof. Lutz Frölich (Mannheim), Prof. Johannes Kornhuber (Erlangen), Prof. Isabella Heuser (Berlin), Prof. Jens Wiltfang (Göttingen), Prof. Otto Rienhoff (Göttingen) und Priv.-Doz. Oliver Peters
(Berlin). Der Vorstand des KND e. V. arbeitet in regelmäßigen Telefonkonferenzen kontinuierlich zusammen und organisiert mindestens einmal jährlich eine Mitgliederversammlung. Der eingetragene Verein KND e. V. verwaltet die Daten- und Biomaterialbanken, koordiniert Publikationsvorhaben und unterstützt fortlaufend Publikationsvorhaben und Anträge auf Bereitstellung von Daten- und Biomaterial von Mitgliedern des Kompetenznetzes, aber auch externe Anfragen. Die Mitglieder des KND e. V. sind nach Auslaufen der Förderung durch das BMBF weiterhin in der Mehrzahl psychiatrische Universitätskliniken, in persona vertreten durch deren Lehrstuhlinhaber oder für den Bereich der klinischen Demenzforschung verantwortliche Ärzte und Wissenschaftler. Die Mitglieder des Kompetenznetzes Demenzen bewirtschaften bis heute aktiv in nationalen und internationalen Kooperationen die im Rahmen der Förderung generierten Daten-und Biomaterialbanken, wodurch auch Jahre nach dem Ende der finanziellen staatlichen Zuwendung eine Fülle von Forschungsarbeiten unterstützt und wissenschaftliche Publikationen ermöglicht werden. Das Kompetenznetz Demenzen e.V. hat eine dauerhafte Struktur nationaler und internationaler wissenschaftlicher Zusammenarbeit geschaffen.

\section{Erfolge während der Förderung}

Mit dem Beginn der Förderung des KND wurde ein einheitliches Schema, wie Gedächtnissprechstunden Patienten mit kognitiven Störungen und demen- ziellen Syndromen untersuchen sollen, festgelegt. Eine solche Festlegung auf Instrumente und Verfahren gab es zuvor nicht, wodurch insbesondere die Vergleichbarkeit der Syndromdiagnose "leichte kognitive Störung" (mild cognitive impairment, $\mathrm{MCI}$ ) nicht gegeben war. Dieses stellte ein wesentliches Hemmnis für die Weiterentwicklung der Frühdiagnose von Demenzerkrankungen dar. Durch das KND wurde eine Festlegung auf eine Batterie von geeigneten neuropsychologischen Testinstrumenten und von verschiedenen Skalen getroffen. Das KND war dadurch Wegbereiter einer Entwicklung, die es erlaubte, geringfügige von erheblichen kognitiven Störungen abzugrenzen, und hat der Festlegung durch etablierte Krankheitsmanuale um Jahre vorgegriffen. Erst im jüngst etablierten Diagnostic and Statistical Manual of Mental Disorders (DSM-V) wurden vergleichbare Festlegungen implementiert („minor" und „major cognitive deficit"), während eine äquivalente Überarbeitung des ICD10-Diagnosesystems (Internationale statistische Klassifikation der Krankheiten und verwandter Gesundheitsprobleme) bislang (noch) nicht erfolgt ist.

Im KND wurde im Rahmen der epidemiologischen Kohortenstudie das Konzept von subjektiven Gedächtnisproblemen (ohne feststellbare objektive kognitive Normabweichung) vorangetrieben. Dieser Ansatz hat nach der neuesten Entwicklung von Biomarkern, die vor der ersten Symptombildung feststellbar sind, in der internationalen Alzheimer-For- 
schung eine wachsende Bedeutung erlangt.

Das KND war auch im Bereich der kranialen Bildgebung mittels Magnetresonanztomographie (MRT) wegbereitend für wichtige Fortschritte auf dem Weg von der einfachen Ausschlussdiagnostik hin zu einer Positivdiagnostik mit prognostischem Wert für den Krankheitsverlauf. Im Rahmen der Bildgebungsprojekte wurden geeignete MRT-Sequenzen für volumetrische Verfahren definiert, die heute essenziell sind für die Beurteilung der Atrophie in verschiedenen Hirnregionen und sowohl differenzialdiagnostischen als auch prognostischen Nutzen haben. Im Bereich der Biomarkerdiagnostik von Proteinen im Blut und Nervenwasser (lumbaler Liquor) wurden Standards für die Abnahme und Weiterverarbeitung von biologischen Proben entwickelt, die Conditio sine qua non für eine standardisierte neurochemische Demenzdiagnostik sind und bis heute in Anschlussprojekten fast unverändert weitere Verwendung finden. Die umfassende biologische Stichprobencharakterisierung erlaubt eine der weltweit ersten erfolgreichen genomweiten Assoziationsuntersuchungen zu den Amyloidmarkern in Liquor mit innovativen Beiträgen zur Pathogenese zur Forschung der Alzheimer-Krankheit.

Unabhängig von den präklinischen und klinisch-wissenschaftlichen Erfolgen hat das Kompetenznetz Demenzen während der Förderung und darüber hinaus, u. a. in Zusammenarbeit mit der Alzheimer Gesellschaft, wesentlich zur Entstigmatisierung von Demenzerkrankungen beigetragen.

\section{Allgemeine strukturelle Leis- tungen - Netzwerkpartizipation}

Neben den konkreten und mittelbaren wissenschaftlichen Errungenschaften ist einer der am schwersten zu fassenden, aber vermutlich größten Verdienste der BMBF-Förderung des Kompetenznetzes Demenzen in der Strukturgebung für die Forschungslandschaft im Bereich Demenzen in Deutschland $\mathrm{zu}$ sehen. Es hat sich über die vergangenen zehn Jahre in einer Vielzahl von kleineren Projekten für die Mitglieder des Kom- petenznetzes Demenzen als äußerst hilfreich erwiesen, über Jahre hinweg mit einer großen Anzahl von akademischen, aber auch nichtakademischen Akteuren (z. B. Alzheimer Gesellschaft) im Bereich demenzielle Erkrankungen zusammengearbeitet zu haben. Bei der Beantragung und Durchführung von Anschlussprojekten war durch die wissenschaftliche Partnerschaft der Grundstein für eine fortgesetzte Zusammenarbeit gelegt.

Kohorten: Das KND hat zwei multizentrisch strukturierte Kohorten aufgebaut:

a) 2200 Patienten mit MCI oder leichtgradigen Demenzen, die klinisch, neuropsychologisch, mit Neuroimaging und bzgl. blut- und liquorbasierten, hypothetischen Biomarkern (inkl. Molekulargenetik) untersucht wurden; nach 2-3 Jahren erfolgte eine klinische Follow-up-Untersuchung. Ziel: Umfassende klinische Charakterisierung von MCI und beginnender Demenz, Evaluation von hypothetischen Biomarkern (z. B. Liquor und Blutmarker, Magnetresonanzspektroskopie) und Prädikation der Konversion zur Demenz.

b) 3300 systematisch rekrutierte Patienten in 120 Hausarztpraxen (diagnostisch nicht vorselektiert, aber ohne Demenz) im Alter von/über 75 Jahren, die klinisch, neuropsychologisch, genetisch und bzgl. ihrer Lebensstile und des Inanspruchnahmeverhaltens untersucht wurden, regelmäßige persönliche Verlaufsuntersuchungen erfolgten im Abstand von jeweils 1,5 Jahren.

AgeCoDe-Kohorte - Ziel: Darstellung der kognitiven Entwicklung im Alter im Zusammenhang mit medizinischen, psychosozialen und lebensstilbezogenen Bedingungen; Entwicklung von Risiko- und Schutzfaktoren sowie Prognosescores für spätere Demenzentwicklung (u. a. auch für die allgemeinärztliche Praxis).

\section{Daten- und Biomaterialbanken}

Während der Förderung durch das BMBF konnten bedeutende Daten- und Bio- materialbanken aufgebaut werden, die bis heute vom KND e. V. weiter unterhalten und bewirtschaftet werden. Die aus zusätzlichen Auswerteprojekten und Messungen an Bilddatensätzen und Biomaterialien gewonnenen Daten werden der bestehenden Datenbank hinzugefügt und ermöglichen neue Korrelationsanalysen. Ein besonders gutes Beispiel hierfür sind die im Verlauf der letzten Jahre verfügbar gewordenen genomweiten Assoziationsstudien(GWAS)-Analysen, die eine Vielzahl von neuen Informationen generiert haben. Durch jüngste Nachfolgeprojekte (BMBF Neuroallianz, siehe nachfolgend im Text) werden neue Daten aus der Langzeitbeobachtung von Probanden und Patienten aus dem Kompetenznetz Demenzen generiert, die die vorhandenen Daten in ihrer Aussagekraft aufwerten und ebenfalls neue Auswertevorhaben und Publikationen ermöglichen werden. Die Datenbanken des KND e. V., bestehend aus einem SecuTrial ${ }^{\circledR}$-System für klinische Daten und einer Bilddatenbank, in welcher kraniale Magnetresonanztomographien gespeichert werden können, werden am Institut für medizinische Informatik in Göttingen (Prof. Otto Rienhoff) verwaltet. Die Biomaterialbanken werden unterhalten an den Universitäten in Erlangen (Prof. Johannes Kornhuber) und in Bonn (Prof. Wolfgang Maier).

\section{Publikationen von besonderer Bedeutung}

Über mehr als zehn Jahre hinweg ist eine Vielzahl von Publikationen, die mittelbar oder unmittelbar aus dem Kompetenznetz hervorgegangen sind, erschienen. Hier soll jeweils eine Arbeit aus den Bereichen Neuropsychologie (E1.1), Biomarkerdiagnostik (E1.2), Bildgebung (E1.3), Therapiestudien (E2), Genetik und Versorgungsforschung (E3) herausgestellt werden.

Neuropsychologie (E1.1). Wagner et al. zeigen, dass ein im KND eingesetztes neuropsychologisches Verfahren, der Free and Cued Selective Reminding Test (FCSRT), eine besonders hohe Korrelation mit den für die AlzheimerErkrankung typischen Biomarkern im 
Liquor zeigt. Der FCSRT ist deshalb sehr geeignet für das Screening im Rahmen von klinischen Studien bei AlzheimerErkrankung im Frühstadium [1].

Wolfsgruber et al. machen in ihrer Arbeit deutlich, dass die individuelle Wahrnehmung von Gedächtnisproblemen einen eigenen Faktor bei der Prognose von Demenzerkrankungen spielt und diese im Rahmen der Frühdiagnostik interpretiert werden muss. In dieser Studie kann gezeigt werden, dass eine hohe Besorgnis und depressive Symptome mit den neurobiologischen Merkmalen der Alzheimer-Erkrankung korrelieren [2].

Biomarkerdignostik (E1.2). In der Arbeit von Wiltfang et al. wird gezeigt, dass die weithin übliche alleinige Bestimmung von einer Sequenz des Amyloidproteins (A $\beta$ 1-42) nicht ausreichend ist, um die Stoffwechsellage dieses für die Alzheimer-Erkrankung zentralen Proteins abzubilden. Die Arbeit weist nach, dass ein Quotient bestehend aus A $\beta$ 142 und $A \beta$ 1-40 deutlich besser mit dem den Grad der Neurodegeneration anzeigenden Tau-Protein korreliert als $A \beta 1$ 42 alleine. Die Ergebnisse dieser Arbeit wurden mittlerweile von anderen Gruppen bestätigt. Die Arbeit trägt mit ihren Ergebnissen essenziell zur Verbesserung der neurochemisch-neurobiologischen Demenzdiagnostik im Liquor bei [3]. Die Erfahrungen mit den etablierten Demenzmarkern im Liquor A $\beta$ 1-42, A $\beta$ 1-40, $t$-Tau und $p$-Tau zeigen, dass dringender Bedarf an zusätzlichen Markern besteht, die einerseits falsch-positive und falsch-negative Ergebnisse vermeiden, andererseits die differenzialdiagnostische Abgrenzung und die Abschätzung der Progression verbessern helfen.

Mit der Arbeit von Lewczuk et al. gelang es, erstmals die Methodik der Messung von löslichem Amyloid-Vorläuferprotein zur Anwendung zu bringen und so für die Verbesserung der neurochemischen Demenzdiagnostik nutzbar zu machen [4].

Bildgebung (E1.3). Die Arbeit von Morgen et al. untersucht den Einfluss von genetischen Markern, verschiedenen Risikogenen für die Alzheimer-Erkrankung,

Bundesgesundheitsbl 2016 59:438-443 DOI 10.1007/s00103-016-2314-y

(c) The Author(s) 2016

O. Peters · I. Heuser $\cdot$ L. Frölich $\cdot$ E. Rüther $\cdot$ O. Rienhoff · J. Kornhuber · J. Wiltfang $\cdot$ W. Maier Das Kompetenznetz Demenzen. Erfolge und Ausblicke

\section{Zusammenfassung}

Hintergrund. Das Kompetenznetz Demenzen (KND) ist ein akademischer Verbund mehrheitlich psychiatrischer Gedächtnissprechstunden und verfolgt drei Ziele: (1) Die Weiterentwicklung der Demenzdiagnostik einschließlich Neuropsychologie, Biomarker, Bildgebung und Genetik. (2) Die Durchführung von Therapiestudien bei kognitiven Störungen und Demenzen. (3) Die Verbesserung der Versorgung von Demenzerkrankten in Deutschland.

Ziel der Arbeit. In diesem Beitrag werden die bisherigen Ergebnisse einzelner Teilprojekte des KND zusammengefasst sowie Ausblicke über die laufende Forschung gegeben. Methoden. Es wurden zwei multizentrische Kohorten aufgebaut. In einer ersten Kohorte wurden Menschen mit einer leichten kognitiven Störung und Menschen mit einer beginnenden Demenz klinisch, neuropsychologisch sowie hinsichtlich verschiedener Biomarker untersucht. Ein Teil dieser Kohorte wurde im Rahmen einer Beobachtungsstudie über Jahre nachverfolgt, ein weiterer Teil im Rahmen von zwei Behandlungsstudien untersucht. In der zweiten Kohorte wurden Menschen ohne Demenz klinisch, neuropsychologisch und genetisch untersucht, und es wurden psychosoziale Faktoren erhoben. In den verschiedenen Teilprojekten des KND werden Daten über Follow-up-Zeiträume bis zu zehn Jahren ausgewertet.

Ergebnisse und Diskussion. Das Kompetenznetz Demenzen hat zahlreiche Publikationen zur Diagnostik und Therapie von kognitiven Störungen und Demenzen sowie zur Versorgung von Demenzerkrankten in Deutschland ermöglicht. Der Verbund hat Strukturen sowie eine Daten- und Biomaterialbank geschaffen, die auch weiterhin die Grundlage für nationale und internationale Kooperationen sind. Darüber hinaus hat das KND zur Entstigmatisierung von Demenzerkrankungen beigetragen.

Schlüsselwörter

Demenz · Leichte kognitive Störung · Biomarker - Bildgebung und Genetik . Medikamentöse Therapiestudien

\section{Dementia Competence Network. Results and outlook}

\section{Abstract}

Background. The Dementia Competence Network (DCN) is represented by academic memory clinics and has three major aims: (1) To facilitate the development of diagnostic tools including neuropsychology, biomarkers, imaging and genetics. (2) To implement clinical trials in mild cognitive impairment and dementia and (3) to improve standard care for dementia in Germany.

Aims. This article summarizes the achievements of the DCN so far and highlights future perspectives.

Methods. The DCN has built up two multicentre cohorts. Within the first cohort, patients with mild cognitive impairment or mild dementia were examined longitudinally using multiple neuropsychological assessments and numerous different biomarkers. In a subgroup of the first cohort, patients were treated with antidementive drugs in two placebo-controlled clinical trials. The second cohort included cognitively healthy older people and examined repetitively clinical, neuropsychological and psychosocial parameters for ten years.

Results and discussion. The DCN has generated a large data and biomaterial bank. Numerous publications have helped to develop further diagnostic procedures and treatment of cognitive disorders and dementia. The DCN has contributed to end stigmatisation of dementia.

\section{Keywords}

Dementia - Mild cognitive impairment . Biomarker - Neuroimaging and genetics . Clinical trials 
auf das Ausmaß der Atrophie und auf die Charakteristik der kognitiven Defizite bei der beginnenden Demenz vom Alzheimer-Typ. Die Arbeit lässt vermuten, dass die Risikogene APOE und PICALM einen Einfluss haben auf die Lokalisation der Hirnatrophie und dieses sich mit entsprechenden kognitiven Defiziten in der Verarbeitungsgeschwindigkeit und beim Arbeitsgedächtnis korrelieren lässt [5].

Therapiestudien (E2). Peters et al. untersuchen die Wirksamkeit und Sicherheit einer Kombinationstherapie bestehend aus dem Acetylcholinesterase-Inhibitor (AChEI) Galantamin-CR und dem NMDA-Rezeptor-Antagonisten $\mathrm{Me}$ mantin. Beide Substanzen sind für die Behandlung der leichten bis mittelschweren (Galantamin) und mittelschweren bis schweren Demenz (Memantin) zugelassen. Es konnte gezeigt werden, dass bei der De-novo-Therapie von vormals unbehandelten Patienten die Kombinationstherapie der Monotherapie nicht überlegen ist. Die Arbeit schafft damit Evidenz für die Handhabung erst bei fortschreitender Erkrankung unter Behandlung mit einem AChEI Memantin zusätzlich oder aber anstelle dessen einzusetzen. Ein weiteres wichtiges Ergebnis besteht darin, in einer kontrollierten Studie gezeigt zu haben, dass die Kombination keine zusätzlichen Nebenwirkungen hat und als sicher in der Anwendung betrachtet werden kann [6].

Epidemiologie und Versorgungsforschung (E3.1 und E3.3). Jessen et al. weisen nach, dass subjektive Gedächtnisbeschwerden die ersten klinisch erkennbaren Zeichen einer AlzheimerErkrankung darstellen; sie sagen auch das spätere Auftreten einer Demenz vom Alzheimer-Typ voraus [7].

Eine (für Deutschland) erste personenbezogene gesundheitsökonomische Analyse zu den verschiedenen Stadien der Erkrankung belegte (auch im internationalen Vergleich) hohe Kosten: zwischen $15.000 €$ (leicht) und $42.000 €$ (schwer), diese Progression resultiert vor allem aus informeller Pflege [8].

Der Zusammenhang subjektiver kognitiver Störungen mit objektivem ko- gnitivem Abbau ist kontrovers diskutiert worden. Durch Growth Curve Modeling der Langzeitdaten aus den Verlaufsuntersuchungen in der AgeCoDe-Kohorte konnte gezeigt werden, dass subjektive Gedächtnisstörungen, insbesondere wenn sie als besorgniserregend bewertet werden, nicht nur spezifisch mit dem Gedächtnisabbau der nachfolgenden Jahre zusammenhängen, sondern dass ihnen ein signifikantes Nachlassen des episodischen Gedächtnisses vorausgeht [9].

Genetik (E 3.2). Die Arbeiten von Heck et al. [10] und Papassotiropoulos et al. [11] zeigen in mehreren Kohorten junger und alter Probanden, dass episodische (hippocampusabhängige) Gedächtnisleistungen mit einer Gruppe von Genvarianten assoziiert sind, die gemeinsam Calciumsignalwege beeinflussen. Interessanterweise sind die gleichen Genvarianten auch mit dem Risiko für die sporadische Alzheimer-Erkrankung assoziiert.

Ramirez et al. charakterisierten die in E1 untersuchte Stichprobe von MCI- und 4D-Patienten genomweit bzgl. der DNASequenzen: Auf Grundlage der Biomarkerdiagnostik (s. oben), konnte das KND (in Zusammenarbeit mit niederländischen und spanischen Arbeitsgruppen) eine der ersten "genomweiten Assoziationsstudien“ zu den Alzheimer-Biomarkern durchführen und genomweit signifikante genetische Marker (ApoE und SUCLG2) erhalten. Da Biomarker den zur Alzheimer-Erkrankung führenden pathogenetischen Prozess abbilden, kommt ihrer genetischen Grundlage besondere Bedeutung zu [12].

\section{Wichtige Folgeprojekte des Kompetenznetzes Demenzen}

Eine Reihe von Nachfolgeprojekten konnte in den Jahren nach Auslaufen der Förderung initiiert werden, entweder als kontinuierliche Nachverfolgung eines Teils der im Rahmen des KND etablierten Kohorten oder aber als Anschlussstudien, welche die im Rahmen des Kompetenznetzes Demenzen aufgebauten Strukturen nutzen. Drei Projekte, die zum gegenwärtigen Zeitpunkt weiterhin aktiv sind, sollen hier kurz skizziert werden:
Neuroallianz-Studie (E1-Nachfolgeprojekt). Unter dem Titel „Multimodale Biomarker zur Vorhersage und zum Staging einer Alzheimer-bedingten demenziellen Entwicklung “ werden „Longterm Follow-up"-Nachuntersuchungen (10-12 Jahre nach Erstuntersuchung) von Probanden mit initial leichter kognitiver Störung durchgeführt, die im Rahmen des Kompetenznetzes Demenzen erstmals unter Zuhilfenahme von Neuropsychometrie, Liquordiagnostik und Bildgebung untersucht wurden. Bei den noch im Rahmen des Kompetenznetzes durchgeführten „Shortterm Follow-up“-Nachuntersuchungen konnten die schnell fortschreitenden Phänotypen der Alzheimer-Erkrankung identifiziert werden. Jetzt werden die langsam fortschreitenden Phänotypen identifiziert mit dem Ziel, ein multimodales Biomarkerprofil zu identifizieren, welches die beiden Gruppen voneinander unterscheiden kann.

SIMaMCI-Studie (E2-Nachfolgeprojekt). Aus epidemiologischen Studien ist bekannt, dass die dauerhafte Einnahme von Cholesterinsenkern mit einem verminderten Risiko, an einer Demenz $\mathrm{zu}$ erkranken, verbunden ist. Cholesterinsenker beeinflussen eine Vielzahl von Mechanismen, die für neurodegenerative Prozesse des Gehirnes verantwortlich sein können. Einige „Proof-ofconcept"-Studien konnten die Wirkung von Cholesterinsenkern auf den Amyloidstoffwechsel im Gehirn zeigen. In der BMBF-geförderten SIMaMCI-Präventionsstudie werden Probanden mit leichter kognitiver Störung und einem erhöhten Risiko, an einer Demenz zu erkranken, mit einem Studienmedikament bestehend aus $60 \mathrm{mg}$ Simvastatin oder Placebo über bis zu vier Jahre (mindestens zwei Jahre) behandelt. Hypothese der Studie ist, dass durch die hochdosierte Einnahme des Cholesterinsenkers Simvastatin der Zeitpunkt des Überschreitens der Schwelle zur Demenz hinausgezögert werden kann. Primärer Outcomeparameter der SIMaMCI-Präventionsstudie ist das „Clinical dementia rating - sum of boxes (CDR)“, mit dem das Fortschreiten von demenzassoziierten Defiziten erfasst wird. 
AgeCoDE-Studie (E3-Nachfolgeprojekt). Die aufgebaute epidemiologische Kohorte AgeCoDE konnte ab 2008, nach Beendigung der KND-Förderung, als Verbund in die nachfolgende BMBFFörderung zu Medizinischen Kompetenznetzen übernommen werden, und zwar im Kompetenznetz Degenerative Demenzen (KNDD). Diese Kohortenstudie befindet sich nach mehreren Verlängerungen und Aufstockungen nun bis 2017 in der BMBF-Förderung. Mehrfach wurden Erweiterungsprojekte über verschiedene BMBF- und BMGProgramme zur Versorgungsforschung finanziert. Damit gehört diese Kohorte zu den am längsten und intensivsten epidemiologisch untersuchten Kohorten in der Altersbevölkerung (mit einer BMBFFörderung über mehr als 15 Jahre); die auf die KND-Förderung aufbauenden internationalen Kooperationen haben sich seit 2008 stark erweitert und haben sich in zahlreichen, teils sehr hochrangigen Publikationen niedergeschlagen.

\section{Unterstützung durch und Zusammenarbeit mit der forschenden Pharmaindustrie}

Die beiden Therapiestudien des Kompetenznetzes Demenzen (E2.1: Kombinationstherapie bei leichter kognitiver Störung und E2.2: Kombinationstherapie bei leichter bis mittelschwerer Demenz vom Alzheimer-Typ) wurden von den Firmen Merz und Janssen-Cilag maßgeblich durch die Bereitstellung der Prüfsubstanzen Galantamin-CR (Reminyl), Memantin (Axura) und Placebo sowie infrastrukturelle Unterstützung gefördert. Beide Firmen haben auch jenseits der Unterstützung des Kompetenznetzes Demenzen in erheblichem Maße zur Aufklärung über und Öffentlichkeitsarbeit für Demenzerkrankungen beigetragen. Zahlreiche Fortbildungen und Informationsschriften für Ärzte und Pflege, aber auch für pflegende Angehörige wurden ermöglicht. Da das Engagement der öffentlichen Hand insbesondere im Bereich der klinischen Demenzforschung nicht ausreichend ist, um die enormen Herausforderungen $\mathrm{zu}$ bewältigen, ist auch zukünftig eine Zusammenarbeit mit der forschenden Pharmaindustrie unabding- bar notwendig. Dass im Rahmen eines öffentlich geförderten Projektes die Wirksamkeit von zugelassenen Medikamenten in der Kombination zu überprüfen möglich ist, konnte im Kompetenznetz gezeigt werden. Diese Zusammenarbeit sollte wegweisend für zukünftige Vorhaben sein.

\section{Internationale Kooperationen}

Die internationalen Kooperationen des KND e. V. wurden in der Mehrzahl über die parallele Mitgliedschaft von Wissenschaftlern im Kompetenznetz Demenzen e. V. und im European Alzheimer's Disease Consortium (EADC) angestoßen. Das EADC organisiert zweimal jährlich ein Meeting von europäischen Alzheimer-Forschern. Im Rahmen dieser Treffen konnten wiederholt gemeinsame Auswerteprojekte, die zu bedeutenden Publikationen oder zu gemeinsamen Antragsstellungen geführt haben, initiiert werden. Für molekulargenetische Studien bestehen Kooperationen mit den Forschungskonsortien: GERAD (The Genetic and Enviromental Risk in Alzheimer's Disease), IGAP (International Genomics of Alzheimer's Project), CHARGE (The Cohorts for Heart and Aging Research in Genomic Epidemiology), PERADES (Defining Genetic, Polygenic and Environmental Risk for Alzheimer's Disease, using multiple powerful cohorts, focussed Epigenetics and Stem cell metabolomics), EADI (European Alzheimer's Disease Initiative).

\section{Perspektiven}

Mitbedingt durch die Charakteristik von chronisch-neurodegenerativen Erkrankungen und deren langsamen Fortschreiten, welches klinisch nur sinnvoll in Langzeitbeobachtungen in großen Verbünden beforscht und letztlich ursächlich entschlüsselt werden kann, hat sich das Kompetenznetz Demenzen mit seinen fortbestehenden Strukturen sowie Daten- und Biomaterialbanken als Glücksfall für die Forschung im Bereich Demenzerkrankungen erwiesen. Die Daten- und Biomaterialbanken des Kompetenznetzes Demenzen e.V. sind nachhaltige Grundlage für aktuelle For- schungsprojekte und Ausgangspunkt für neue Vorhaben. Bis dato gelingt es dem Kompetenznetz Demenzen e.V. auch Jahre nach Auslaufen der Förderung, die erarbeiteten Ressourcen für neue Erkenntnisse im Bereich der Demenzforschung in auch weiterhin fortlaufenden Publikationen zu nutzen. Mit dieser Perspektive wird das Kompetenznetz Demenzen e. V. auch in den kommenden Jahren weiterarbeiten.

\section{Korrespondenzadresse}

\section{Priv.-Doz. Dr. O. Peters}

Klinik für Psychiatrie und Psychotherapie, Charité Universitätsmedizin Berlin - CBF Berlin, Deutschland oliver.peters@charite.de

\section{Einhaltung ethischer Richtlinien}

Interessenkonflikt. O. Peters, I. Heuser, L. Frölich E. Rüther, O. Rienhoff, J. Kornhuber, J. Wiltfang und W. Maier geben an, dass kein Interessenkonflikt besteht.

Die in dieser Übersichtsarbeit durchgeführten klinischen Studien wurden von den lokalen Ethikkommissionen der teilnehmenden Universitäten überprüft und genehmigt. Die Arbeit des Kompetenznetz Demenzen wurde vom Bundesministerium für Bildung und Forschung gefördert (BMBF; Förderkennzeichen 01GI0420).

Open Access. This article is distributed under the terms of the Creative Commons Attribution 4.0 International License (http://creativecommons.org/ licenses/by/4.0/), which permits unrestricted use, distribution, and reproduction in any medium, provided you give appropriate credit to the original author(s) and the source, provide a link to the Creative Commons license, and indicate if changes were made.

\section{Literatur}

1. Wagner M, Wolf S, Reischies FM (2012) Biomarker validation of a cued recall memory deficit in prodromal Alzheimer disease. Neurology 78(6):379-386

2. Wolfsgruber S, Jessen F, Koppara A (2015) Subjective cognitive decline is related to CSF biomarkers of $A D$ in patients with $\mathrm{MCl}$. Neurology 84(12):1261-1268

3. Wiltfang J, Esselmann $\mathrm{H}$, Bibl $\mathrm{M}$ et al (2007) Amyloid beta peptide ratio $42 / 40$ but not $A$ beta 42 correlates with phospho-Tau in patients with low- and high-CSF A beta 40 load. J Neurochem 101(4):1053-1059

4. Lewczuk $P$, Kamrowski-Kruck $\mathrm{H}$, Peters $\mathrm{O}$ et al (2010) Soluble amyloid precursor proteins in the cerebrospinal fluid as novel potential biomarkers of Alzheimer's disease: a multicenter study. Mol Psychiatry 15(2):138-145

5. Morgen K, Ramirez A, Frölich L et al (2014) Genetic interaction of PICALM and APOE is associated 
with brain atrophy and cognitive impairment in Alzheimer's disease. Alzheimers Dement 10(5 Suppl):269-276

6. Peters O, Fuentes M, Joachim LK et al (2015) Combined treatment with memantine and galantamine-CR compared with galantamine-CR only in anti-dementia drug naïve patients with mild to moderate Alzheimer's disease. Alzheimers Dement 1(3):198-204

7. Jessen F, Wiese B, Bachmann C et al (2012) Prediction of dementia by subjective memory impairment. Arch Gen Psychiatry 67(4):414-422

8. Leicht $\mathrm{H}$, Heinrich S, Heider D et al (2011) Net costs of dementia by disease stage. Acta Psych Scand 124(5):384-395

9. Koppara A, Wagner M, Lange C et al (2015) Cognitive performance before and after the onset of subjective cognitive decline in old age. Alzheimers Dement 1:194-205

10. Heck A, Fastenrath M, Coynel D et al (2015) Genetic analysis of association between calcium signaling and Hippocampal activation, memory performance in the young and old, and risk for sporadic alzheimer disease. JAMA Psychiatry 72(10):1029-1036

11. Papassotiropoulos A, Henke K, Stefanovaet E et al (2011) A genome-wide survey of human shortterm memory. Mol Psychiatry 16:184-192

12. Ramirez A, Van der Flier WM, Herold C et al (2014) SUCLG2 indentified as both a determinator of CSF Aß1-42 levels and an attenuator of cognitive decline in Alzheimer's disease. Hum Mol Genet 23:6644-6658 